\title{
Symbiotic Simulation Control in Semiconductor Manufacturing
}

\author{
Heiko Aydt ${ }^{1}$, Stephen John Turner ${ }^{1}$, Wentong Cai ${ }^{1}$, Malcolm Yoke Hean Low ${ }^{1}$, \\ Peter Lendermann ${ }^{2}$, and Boon Ping Gan ${ }^{2}$ \\ ${ }^{1}$ School of Computer Engineering, Nanyang Technological University, \\ Singapore 639798 \\ \{aydt, ass jturner, aswtcai, yhlow\}@ntu.edu.sg \\ ${ }^{2}$ D-SIMLAB Technologies Pte Ltd, 30 Biopolis Street \#09-02, Matrix, \\ Singapore 138671 \\ \{peter, boonping\}@d-simlab.com
}

\begin{abstract}
Semiconductor manufacturing is a highly complex and asset intensive process. Solutions are needed to automate control of equipment and improve efficiency. We describe a symbiotic simulation control system which uses reactive what-if analysis to find a stable configuration of a wet bench tool set. This control system is based on a generic framework for symbiotic simulation. We show that symbiotic simulation can be used to make control decisions in near real-time. Furthermore, we show that our approach yields a notable performance improvement over common practise.
\end{abstract}

\section{Introduction}

Semiconductor manufacturing is a complex process which turns pure silicon wafers into integrated circuits with thousands of components. The whole process may require up to several hundred processing steps and up to three months for production [1. One crucial factor for competitiveness in semiconductor manufacturing is ongoing improvement of the manufacturing process 1]. Automation is a critical factor in this context and the semiconductor industry invests several million US dollars for solutions. According to [2, investments for integrated automation solutions can range between US\$130 million and US\$ 180 million. These integrated solutions also include systems for real-time control of equipment. In addition to high complexity, the semiconductor manufacturing process is also an asset intensive process. A variety of tools are used in semiconductor manufacturing to process wafers and a single tool can cost up to US\$2 million 3]. Improvement of tool efficiency is therefore important in order to reduce cost for additional tools.

Several performance metrics are considered in semiconductor manufacturing, of which throughput, cycle time, and on-time delivery are considered the most important ones 4]. The performance of most tools depends on their operation mode, further referred to as configuration, and the distribution of different products, further referred to as product mix. If the product mix is changing, it might 
be necessary to reconfigure various tools in order to maintain performance targets. Decisions regarding this are currently made by engineers who estimate the required capacity for each product mix. Decisions are then implemented by workers who manually change the configuration of a tool. However, workers do not always strictly follow their instructions. This, and the fact that decision making relies mostly on the experience of engineers, causes the demand for an automated control solution.

Simulations have already been used for offline analysis and optimisation of parameters in the manufacturing process. However, on-line solutions which use simulation for real-time control are still in their infancy. Symbiotic simulation, which is driven by sensor data and capable of making control decisions in realtime, seems to be a promising solution for automation and to improve equipment efficiency. In this paper, we show how a dynamic data driven application can be used for operational decision making regarding the configuration of a wet bench toolset (WBTS), which is used for one of the many critical operations in semiconductor manufacturing. We describe a symbiotic simulation control system $(S S C S)$ which is based on a generic framework for symbiotic simulation being developed by us. The application of symbiotic simulation in semiconductor manufacturing also represents the first show-case for the generic framework. The need for such a generic symbiotic framework has been articulated in [5].

The paper is structured as follows: Before presenting our own solution, we give an overview of related work in Sect. 2. This is followed by an introduction to the SSCS and a brief overview of the generic framework in Sect. 3 . A more detailed description of the WBTS is given in Sect. 4. We compare common practise with our approach of controlling the WBTS. Therefore, we explain both approaches in Sect. 5 before presenting our experimental results in Sect. 6. Our conclusions are given in Sect. 7 .

\section{Related Work}

The term symbiotic simulation was coined at the Dagstuhl Seminar on Grand Challenges for Modeling and Simulation in 2002 [6]. Symbiotic simulation is closely related to dynamic data-driven application systems (DDDAS). While DDDAS emphasises the symbiotic relationship between an application and a measurement process, symbiotic simulation rather focuses on the symbiotic relationship between a simulation and a physical system. However, symbiotic simulation is also dynamic data-driven and may use sensor steering to improve the accuracy of simulations. Several applications which use symbiotic simulation have been developed since 2002. These include the application of symbiotic simulation for UAV path planning [7], social sciences [8], 9], and business process re-engineering [10. In the context of semiconductor manufacturing, symbiotic simulation has already been used in 11] to optimise backend operations.

Simulations have been used in several ways to improve the performance of a semiconductor manufacturing process. Efforts in this context mainly aim to improve the cycle time. For example, in 3. a two day reduction of total cycle 
time could be achieved by investigating a real wafer factory based on simulations. Other work which is concerned with improving cycle times can be found in 12, 13, 14. Wet bench tools have been the subject of various works. Lee et al. [15] examine the scheduling problem of a wet station for wafer cleaning using Petri net modelling and Gan et al. [16] evaluate the effect of upgrading a bottleneck furnace to the wet bench performance with increasing wafer demand.

\section{Symbiotic Simulation Control System (SSCS)}

An SSCS is a specific class of symbiotic simulation system. It makes decisions based on the simulation and evaluation of what-if scenarios, each representing an alternative decision, and implements them by means of actuators which are available to the system. A reactive what-if analysis aims to find immediate solutions to sudden, and potentially critical, conditions. For example, the performance of a manufacturing system is observed and a reactive what-if analysis is carried out once the performance drops below a predefined value, i.e., once the performance becomes critical. Various what-if scenarios, each representing alternative decision options (e.g., tool configurations), are simulated and analysed. The decision option of the scenario which performs best in simulation is implemented.

The SSCS is realised using an implementation of our generic framework for symbiotic simulation systems. This framework is based on Jadex 1, a BDI agent system, and represents a library of functional components which are typically required in a symbiotic simulation system. For example, creating and simulating alternative what-if scenarios is an essential concept in symbiotic simulation. Each of these functionalities, among several others, is realised as functional component and provided by the framework.

We use capabilities [17, 18, to implement the various functional components of the framework. The concept of capabilities is used in BDI agent systems to facilitate modularisation of functionality. Agents can be equipped with an arbitrary number of capabilities. This allows flexible design of application-specific symbiotic simulation systems. For example, the design of inherently distributed applications may involve several agents, of which each of them is equipped with only one capability. On the other hand, it is possible to design a symbiotic simulation system which is represented by a single agent only. In this case, the agent is equipped with all required capabilities. The use of capabilities also allows the design of tailor-made symbiotic simulation systems by using only those capabilities which are required in the context of the application.

There are a number of distinct capabilities which are required to realise a symbiotic simulation system. The following description of the SSCS includes only those which are used in the context of the WBTS application. An overview of the WBTS-SSCS capabilities is illustrated in Fig. 1]

The architecture of a symbiotic simulation system consists of three layers for perception, processing, and actuation. The perception layer is responsible for providing measured data to the processing layer where what-if analysis is carried

\footnotetext{
1 http://vsis-www.informatik.uni-hamburg.de/projects/jadex/
} 


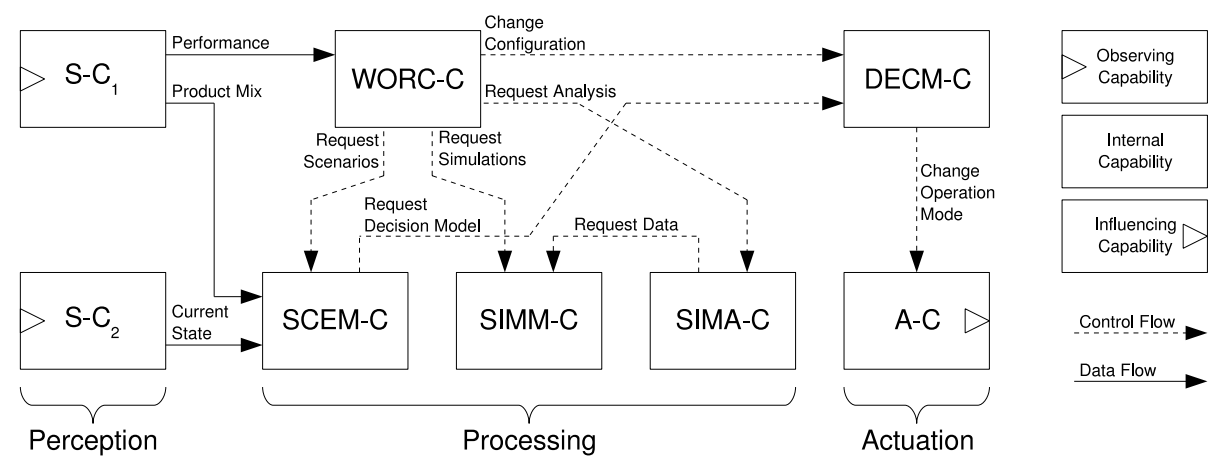

Fig. 1. Overview of various capabilities in the WTBS-SSCS

out. Once a decision is made, the actuation layer is responsible for implementing it. Each layer is associated with a number of specific capabilities.

The workflow control capability WORC-C is the heart of the SSCS. It is responsible for interpreting performance measurements provided by sensor capability $\mathrm{S}_{-} \mathrm{C}_{1}$, triggering the what-if analysis process if the performance becomes critical, and making control decisions regarding the configuration of wet bench tools. If a what-if analysis is triggered, the WORC-C requests a set of scenarios from the scenario management capability SCEM-C, invokes simulations using the simulation management capability SIMM-C, and interprets analysis results provided by the simulation analysis capability SIMA-C. Based on these results, the best decision option is determined by the WORC-C and implemented by using the decision management capability DECM-C.

An SSCS can only implement decision options which are supported by actuators that are available to the SSCS. A decision model is used to reflect possible control options, depending on the available actuators and their abilities to control different aspects of the physical system. In the context of the WBTS application, the decision model reflects the possible operation modes for each wet bench tool. The DECM-C keeps track of available actuators and updates the decision model accordingly.

When requested by the WORC-C, the SCEM-C creates a number of scenarios based on the decision model. Each scenario reflects a different decision option, i.e., a different wet bench configuration. A scenario also consists of an initial state for the simulation run. This initial state is the current state of the physical system, provided by $\mathrm{S}_{-} \mathrm{C}_{2}$. The state of the system represents a snapshot of the WBTS including all wafer lots which are currently being processed. In addition to the decision option and the initial state, a scenario also consists of a given product mix which is used to drive the simulation. The current product mix is provided by $\mathrm{S}-\mathrm{C}_{1}$.

Sensors, actuators, and simulators are inherently application specific. Therefore, the framework cannot provide generic solutions for their corresponding 
capabilities. Instead, application specific implementations have to be provided by the application developer. The framework provides generic solutions for all other capabilities. However, this does not rule out the possibility of using application specific implementations instead of generic standard solutions.

\section{Wet Bench Tool Set (WBTS)}

In semiconductor manufacturing, a wet bench is used to clean wafers after certain fabrication steps to yield higher wafer reliability [15, [19]. A wet bench tool consists of a number of baths which contain chemical liquids and process wafer lots according to recipes. Typically, a wafer fab operates a number of wet benches with different bath setups to support a variety of recipes. A recipe specifies constraints regarding the sequence and timing for the cleaning process. Each lot is associated with a recipe and has to be processed strictly according to it. Neither the sequence of chemical liquids in which a lot is processed, nor the time each wafer spends in a particular chemical liquid must be changed. Violation of these requirements would lead to significantly reduced yield.

Particles of contaminants are introduced into the baths during the cleaning process. Therefore, the chemical liquids have to be changed after a certain time. Some recipes introduce particles faster than others. We therefore distinguish between 'dirty' and 'clean' recipes [16]. In order not to compromise the quality of wafers associated with clean recipes, wafers associated with dirty recipes are processed by separate wet benches. Thus, a wet bench operates either in 'clean' mode or 'dirty' mode. By operation mode we mean that a wet bench is dedicated to either one of the two recipe types. Switching the operation mode from 'clean' to 'dirty' does not require any particular activity. However, switching from 'dirty' to 'clean' requires a complete change of liquids. This activity takes up to several hours [16].

\section{Control Approaches}

\subsection{Common Practise Approach}

The common practise approach involves engineers who decide upon reconfiguration of wet bench tools. Decision making depends mostly on experience and is therefore difficult to capture. With information from the semiconductor industry we developed the following heuristic: 1) If the number of pending wafer lots exceeds 40, the critical recipe (the most frequent recipe) is determined. 2) In the next step, the set of wet benches is identified which is capable of processing the critical recipe but is not configured to do so yet. 3) One of the wet benches is selected and reconfigured accordingly. This is due to the fact that engineers tend to be careful and change only one wet bench at a time. 4) After changing the operation mode, the system is allowed some time to settle. 5) If, after the settling-in time, the situation did not improve the process is repeated. 
We assume a settling-in time of one hour when the operation mode is switched from 'clean' to 'dirty' and four hours otherwise. The latter takes account for a three hour maintenance process where chemicals are changed.

\subsection{SSCS Approach}

Similar to the common practise approach, the WORC-C of the SSCS observes the number of pending wafer lots. If this number exceeds 40, a reactive whatif analysis is triggered. Each what-if scenario reflects the current state of the physical system with an alternative wet bench configuration. In this particular application, we use eight wet bench tools, of which each can operate either in 'clean' or 'dirty' mode. This results in a total of 256 different possible scenarios, created by the SCEM-C. The performance for the next 24 hours is simulated by the SIMM-C for each scenario. The SIMA-C analyses the simulation results and determines the average performance of each scenario in terms of throughput. The best configuration is determined by the WORC-C and implemented by the DECM-C. To implement a decision, the DECM-C sends corresponding control signals to the A-C, which is capable of changing the operation modes of wet benches in the physical system. Once a decision is implemented, the SSCS is inactive for a fixed settling-in period of four hours.

\section{Experiments}

For practical reasons, experiments were not performed with a real physical system. Instead, we used a simulation of the WBTS to emulate the physical system. The emulator was paced at a speed 3600 times faster than real-time. This enabled us to perform experiments, reflecting a period of several months, within a few hours. We also used a mechanism to create snapshots of the emulator at any time. Such a snapshot can then be used as initial state for the simulation of a what-if scenario.

The simulation model is deterministic with the only exception being the lot generating process. This process generates arrival events of wafer lots with a distribution according to a given product mix. The SSCS has to consider this when performing a what-if analysis. Therefore, each what-if scenario is simulated using five replications. A whole what-if analysis step, including scenario creation, simulation, analysis, and decision propagation, takes approximately 50-60 seconds on a cluster with 60 processors.

Each experiment was repeated ten times and the mean values over all results are presented here. All results show the performance of the WBTS in terms of cycle time. The cycle time is determined by the ratio of the overall time a wafer lot spent at the toolset to the processing time according to the associated recipe. The overall time includes the time a wafer lot has to wait before being processed. 


\subsection{Alternating Product Mixes}

With the first experiment, we aim to show how well both approaches can maintain performance with an alternating product mix. We use two product mixes with a constant load of 900 lots per day. In this experiment, a period of 20 weeks is considered. Within this period, the product mix is changed every two weeks. We choose the product mixes so that there exists no configuration which is stable for both. This means, a configuration which is stable assuming one of the product mixes, is unstable when assuming the other product mix. An unstable configuration results in a throughput which is below the load. As a consequence, queues of pending lots will build up and an increasing cycle time can be observed. To avoid this, the wet bench configuration has to be changed eventually. Figure 2 illustrates the performance of the SSCS approach and the common practise approach.
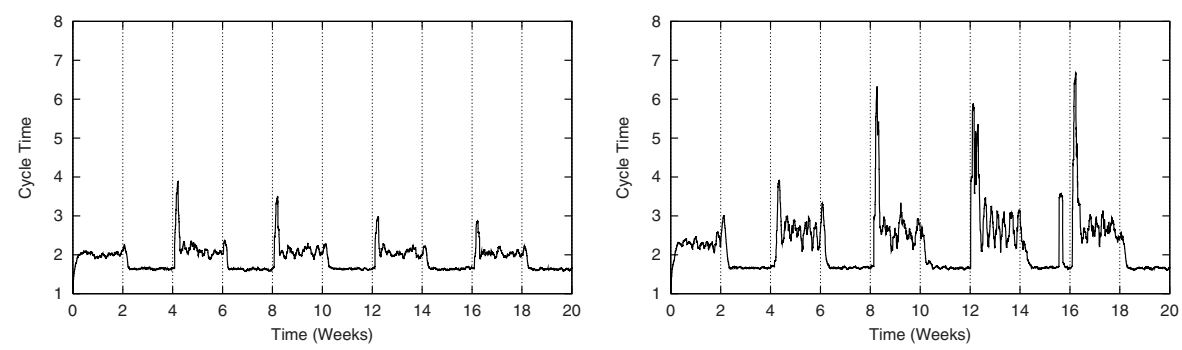

Fig. 2. Cycle time of the WBTS over a period of 20 weeks using the SSCS (left) and the common practise approach (right)

In general, the SSCS approach produces a more homogeneous performance with less variability and lower mean cycle time $\left(S_{S S C S}=0.30, \bar{X}_{S S C S}=1.89\right)$ than the common practise approach $\left(S_{C P}=0.76, \bar{X}_{C P}=2.26\right)$. One major disadvantage of the common practise approach is the fact that it is a trial and error approach which changes only one tool configuration at a time. Therefore, it takes longer to find a stable configuration, if at all. On the contrary the SSCS is capable of finding a stable solution, if there is one, and making all necessary changes in one step. If no stable solution exists, the solution which performed best in simulations is applied.

\subsection{High Workload}

At increasing load, it is necessary to oscillate between configurations. This is due to the fact that only two of the eight wet benches used in this application have a bath setup which allows them to process a large variety of recipes. All other wet benches have setups which are limited to a smaller group of recipes. Therefore, these two wet benches represent a bottleneck at high loads. To overcome this, it is necessary to switch the configurations of these wet benches back and forth. 
As a consequence, the performance is stabilised. However, above a certain load, the performance cannot be stabilised anymore.

The second experiment aims to determine the critical load of both approaches. The SSCS approach, which is more sophisticated, is capable of stabilising the performance at higher loads than the common practise approach. Figure 3 and 4 illustrate the performance of both approaches over a period of one month with loads of 1000/1200 and 1500/1700 lots per day, respectively.
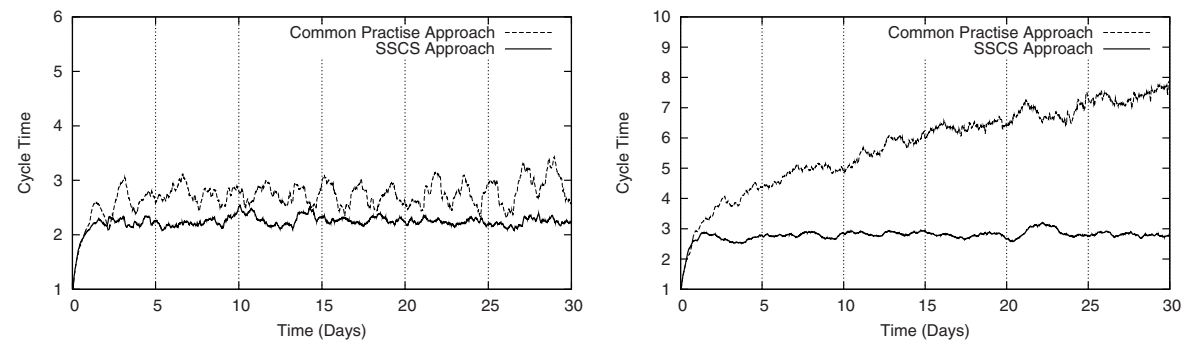

Fig. 3. Cycle time of the WBTS over a period of one month using a load of 1000 (left) and 1200 (right) lots per day

Both approaches are capable of handling a load of 1000 lots per day. At a load of 1200 lots per day, the performance becomes unstable when using the common practise approach, i.e., the cycle time is constantly increasing. In contrast, the SSCS is still capable of maintaining a stable mean cycle time.
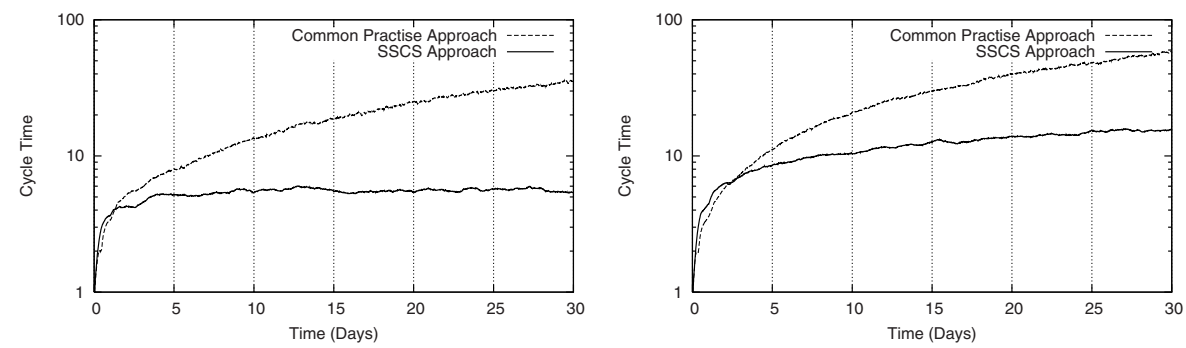

Fig. 4. Cycle time of the WBTS over a period of one month using a load of 1500 (left) and 1700 (right) lots per day. A logarithmic scale was used for cycle time values.

The mean cycle time is elevated at a load of 1500 lots per day but the SSCS approach is still able to handle it. However, at a load of 1700 lots per day the performance finally becomes unstable.

The critical load for the common practise approach and the SSCS approach is at approximately 1000-1200 and 1500-1700 lots per day, respectively. This indicates that the SSCS allows the use of loads which are 25-70\% higher as compared to the common practise approach. 


\section{Conclusions}

In this paper, we have described how a DDDAS, in particular symbiotic simulation, can be used for real-time control of semiconductor equipment and to improve performance. Our experimental results show that using the SSCS yields a notable performance improvement over common practise. This is important as higher efficiency reduces demand for additional equipment. Decision making is performed in reasonably short time. All this indicates the applicability of symbiotic simulation in the context of integrated automation solutions. In future work, we will investigate how an SSCS can be applied to an entire semiconductor factory. This will also include investigation of different forms of what-if analysis.

\section{References}

1. Potoradi, J., Boon, O., Fowler, J., Pfund, M., Mason, S.: Using simulation-based scheduling to maximize demand fulfillment in a semiconductor assembly facility. In: Proceedings of the Winter Simulation Conference, pp. 1857-1861 (2002)

2. Gan, B.P., Chan, L.P., Turner, S.J.: Interoperating simulations of automatic material handling systems and manufacturing processes. In: Proceedings of the Winter Simulation Conference, pp. 1129-1135 (2006)

3. Scholl, W., Domaschke, J.: Implementation of modeling and simulation in semiconductor wafer fabrication with time constraints between wet etch and furnace operations. In: IEEE Transactions on Semiconductor Manufacturing, August 2000, vol. 13, pp. 273-277 (2000)

4. Pfund, M.E., Mason, S.J., Fowler, J.W.: Semiconductor Manufacturing Scheduling and Dispatching. In: Handbook of Production Scheduling. International Series in Operations Research \& Management Science, vol. 89, pp. 213-241. Springer, New York (2006)

5. Huang, S.Y., Cai, W., Turner, S., Hsu, W.J., Zhou, S., Low, M.Y.H., Fujimoto, R., Ayani, R.: A generic symbiotic simulation framework. In: Proceedings of the 20th Workshop on Principles of Advanced and Distributed Simulation, pp. 131-131 (2006)

6. Fujimoto, R., Lunceford, D., Page, E. (eds.): A.M.U.: Grand challenges for modeling and simulation: Dagstuhl report. Technical Report 350, Schloss Dagstuhl. Seminar No 02351 (August 2002)

7. Kamrani, F., Ayani, R.: Using on-line simulation for adaptive path planning of UAVs. In: Proceedings of the 11th IEEE International Symposium on Distributed Simulation and Real-time Applications, Chania, Greece, October 2007, pp. 167-174 (2007)

8. Kennedy, C., Theodoropoulos, G.K., Sorge, V., Ferrari, E., Lee, P., Skelcher, C.: AIMSS: An architecture for data driven simulations in the social sciences. In: Shi, Y., van Albada, G.D., Dongarra, J., Sloot, P.M.A. (eds.) ICCS 2007. LNCS, vol. 4487, pp. 1098-1105. Springer, Heidelberg (2007)

9. Kennedy, C., Theodoropoulos, G.K.: Intelligent management of data driven simulations to support model building in the social sciences. In: Alexandrov, V.N., van Albada, G.D., Sloot, P.M.A., Dongarra, J. (eds.) ICCS 2006. LNCS, vol. 3993, pp. 562-569. Springer, Heidelberg (2006) 
10. Low, M.Y.H., Turner, S.J., Ling, D., Peng, H.L., Chai, L.P., Lendermann, P., Buckley, S.: Symbiotic simulation for business process re-engineering in high-tech manufacturing and service networks. In: Proceedings of the Winter Simulation Conference (2007)

11. Low, M.Y.H., Lye, K.W., Lendermann, P., Turner, S.J., Chim, R.T.W., Leo, S.H.: An agent-based approach for managing symbiotic simulation of semiconductor assembly and test operation. In: Proceedings of the 4th International Joint Conference on Autonomous Agents and Multiagent Systems, pp. 85-92. ACM Press, New York (2005)

12. Rulkens, H., van Campen, E., van Herk, J., Rooda, J.: Batch size optimization of a furnace and pre-clean area by using dynamic simulations. In: Advanced Semiconductor Manufacturing Conference and Workshop (IEEE/SEMI), Boston, MA, USA, September 1998, pp. 439-444 (1998)

13. Potoradi, J., Winz, G., Kam, L.W.: Determining optimal lot-size for a semiconductor back-end factory. In: Proceedings of the Winter Simulation Conference, pp. 720-726 (1999)

14. Akçali, E., Uzsoy, R., Hiscock, D.G., Moser, A.L., Teyner, T.J.: Alternative loading and dispatching policies for furnace operations in semiconductor manufacturing: a comparison by simulation. In: Proceedings of the Winter Simulation Conference, pp. 1428-1435 (2000)

15. Lee, T.E., Lee, H.Y., Lee, S.J.: Scheduling a wet station for wafer cleaning with multiple job flows and multiple wafer-handling robots. International Journal of Production Research 45, 487-507 (2007)

16. Gan, B.P., Lendermann, P., Quek, K.P.T., van der Heijden, B., Chin, C.C., Koh, C.Y.: Simulation analysis on the impact of furnace batch size increase in a deposition loop. In: Perrone, L.F., Lawson, B., Liu, J., Wieland, F.P. (eds.) Proceedings of the Winter Simulation Conference, pp. 1821-1828 (2006)

17. Busetta, P., Howden, N., Rönnquist, R., Hodgson, A.: Structuring BDI agents in functional clusters. In: Jennings, N.R. (ed.) ATAL 1999. LNCS, vol. 1757, pp. 277-289. Springer, Heidelberg (2000)

18. Braubach, L., Pokahr, A., Lamersdorf, W.: Extending the capability concept for flexible BDI agent modularization. In: Bordini, R.H., Dastani, M., Dix, J., Seghrouchni, A.E.F. (eds.) PROMAS 2005. LNCS (LNAI), vol. 3862, pp. 139-155. Springer, Heidelberg (2006)

19. Lu, J.K., Ko, F.H., Chu, T.C., Sun, Y.C., Wang, M.Y., Wang, T.K.: Evaluation of cleaning efficiency with a radioactive tracer and development of a microwave digestion method for semiconductor processes. Analytica Chimica Acta 407, 291300 (2000) 